\title{
Clinical significance of ovarian stromal blood flow in assessment of ovarian response in stimulated cycle for in vitro fertilization
}

\author{
Aashima Arora, Shalini Gainder*, Lakhbir Dhaliwal, Vanita Suri
}

Department of Obstetrics and Gynaecology, Post Graduate Institute of Medical Education and Research, Sector 12, Chandigarh, India

Received: 22 July 2015

Accepted: 14 August 2015

\section{*Correspondence:}

Dr. Shalini Gainder,

E-mail: shalinilakhanpal@ @ediffmail.com

Copyright: (c) the author(s), publisher and licensee Medip Academy. This is an open-access article distributed under the terms of the Creative Commons Attribution Non-Commercial License, which permits unrestricted non-commercial use, distribution, and reproduction in any medium, provided the original work is properly cited.

\begin{abstract}
Background: Several ultrasound parameters have been examined to predict the ovarian response to gonadotropins. The only parameter that has been conclusively proven to predict ovarian response is the antral follicle count. It has been suggested that ovarian blood flow may play a crucial role in the development of ovarian follicles. The present study was planned to prospectively analyse the effect of ovarian stromal blood flow on ovarian responsiveness in terms of number of oocytes retrieved in stimulated cycle for IVF.

Methods: Fifty subjects between 22 to 37 years of age with male factor, tubal factor or unexplained infertility underwent controlled ovarian stimulation by the standard "Long protocol". Ovarian stromal blood flow was recorded on baseline transvaginal sonography on day 2 or 3 of cycle and was correlated with number of oocytes retrieved in IVF cycle.

Results: The ovarian stromal blood flow was found to be negatively correlated with age. The number of follicles formed in a subject after stimulation with gonadotropins was negatively correlated with ovarian stromal resistance index and pulsatility index.

Conclusions: Ovarian blood flow predicts ovarian responsiveness and hence provides a non-invasive and cost effective prognostic factor of IVF outcome.
\end{abstract}

Keywords: Ovarian stromal blood flow, IVF outcome

\section{INTRODUCTION}

About 8 to $12 \%$ of all couples worldwide are or have been infertile. Assisted Reproductive Techniques, such as controlled ovarian stimulation and in-vitro fertilization (IVF), are helping many couples with tubal factor, male factor or unexplained infertility to become pregnant. However, despite many advances in IVF techniques, only about 25-30 \% IVF treatment cycles lead to a pregnancy. ${ }^{1}$

The ability of the ovary to respond to exogenous gonadotropin stimulation and to produce several mature oocytes simultaneously is essential for successful IVF.
Several ultrasound parameters have been examined to predict the ovarian response to gonadotropins. The only parameter that has been conclusively proven to predict ovarian response is the antral follicle count (AFC). It was emphasized by Chang et al, NgEHY et al and Hseih YY et al that the response to ovarian stimulation could be determined using the initial follicle count. ${ }^{2-4}$

It has been suggested that ovarian blood flow may play a crucial role in the development of ovarian follicles. Better stromal blood flow may lead to a greater delivery of gonadotropins to the granulosa cells while the perifollicular blood flow may be capable of influencing 
and mediating oocyte maturation, its potential ability to fertilize and develop, as well as oocyte quality. The present study was planned to prospectively analyse the effect of ovarian stromal blood flow on ovarian responsiveness in terms of number of oocytes retrieved in stimulated cycle for IVF.

\section{METHODS}

Fifty couples recruited for IVF in the infertility clinic of a tertiary care research hospital were included in this study. Written consent was obtained from the subjects; also clearance from the Ethics Committee of the institute was obtained. The study included subjects between 22 and 37 years of age with normal baseline LH and FSH levels with cause of infertility being one or more of male factor, tubal factor or unexplained infertility. Patients with any endocrinal imbalance (hypothyroidism, hyperprolactinemia, hypogonadotropic hypogonadism) or a history of ovarian surgery were excluded from this study.

All transvaginal sonographic examinations were performed by the same experienced operator using a 9.5 $\mathrm{MHz}$ probe for $\mathrm{B}$ - Mode and color imaging as well as pulsed Doppler spectral analysis. All scans were performed between 8:00 am to 10:00 am in a quiet and comfortable location after rest of 20 minutes to all subjects.

All subjects underwent a baseline transvaginal sonography on day 2 or day 3 of the cycle and morphology of uterus and both ovaries was noted. The ovarian stromal blood flow was also recorded in the form of Resistance Index (RI) and Pulsatility Index (PI). To record the RI and PI of the ovarian stromal blood flow, the arteries within the ovarian stroma were visualized using the Power Doppler technique, ensuring that no perifollicular blood flow was included. The Doppler gate was then positioned over the ovarian stromal vessels and the flow velocity waveforms were traced until at least three waveforms with similar amplitude occurred. A computer generated auto calculation box was positioned at this site and the RI and PI were calculated.

\section{Statistical analysis}

Continuous variables were analyzed using either the unpaired Student's t-test (normal data distribution) or Mann Whitney $U$ test (skewed data) to compare two means. The data was tested for normal distribution using Kolmogorov-Smirnov (K-S) test. Categorical variables were analyzed using the Chi-square $(\chi 2)$ test. Pearson's correlation coefficient was used to assess significant correlation between variables. p-value $<0.05$ was considered statistically significant. Statistical analysis was performed using Statistical Package for Social Sciences (SPSS) version 11.0.

\section{RESULTS}

A total of 50 subjects who underwent IVF using the standard long stimulation protocol were studied. The age of patients and the type, duration and cause of infertility are depicted in Table 1.

Table 1: Demographic profile of patients.

\begin{tabular}{|llll|} 
Characteristic & Distribution & $\begin{array}{l}\text { Number } \\
\text { of } \\
\text { patients }\end{array}$ & $\begin{array}{l}\text { Perce } \\
\text { ntage }\end{array}$ \\
\hline Age (years) & $22-25$ & 1 & 2 \\
\hline & $26-30$ & 10 & 20 \\
\hline & $31-35$ & 24 & 48 \\
\hline & $>35$ & 15 & 30 \\
\hline $\begin{array}{l}\text { Type of } \\
\text { infertility }\end{array}$ & Primary & 34 & 68 \\
\hline & Secondary & 16 & 32 \\
\hline $\begin{array}{l}\text { Duration of } \\
\text { infertility } \\
\text { (months) }\end{array}$ & $<48$ & 8 & 16 \\
\hline & $48-96$ & 19 & 38 \\
\hline & $>96$ & 23 & 46 \\
\hline $\begin{array}{l}\text { Cause of } \\
\text { infertility }\end{array}$ & Tubal factor & 33 & 66 \\
\hline & Unexplained & 17 & 34 \\
\hline
\end{tabular}

The baseline ultrasound was done on day 2 or 3 of the menstrual cycle in every subject and the ovarian stromal blood flow was noted. The blood flow in both the two ovaries were compared using paired t-test which depicted that the difference between the Resistance Indices and the Pulsatility Indices of the two ovaries of a particular subject was not statistically significant ( $p=0.22$ and 0.24$)$. As there were no statistically significant differences in the Doppler measurements between the right and left ovaries of an individual; the mean RI and PI were calculated for each patient and used for subsequent analysis.

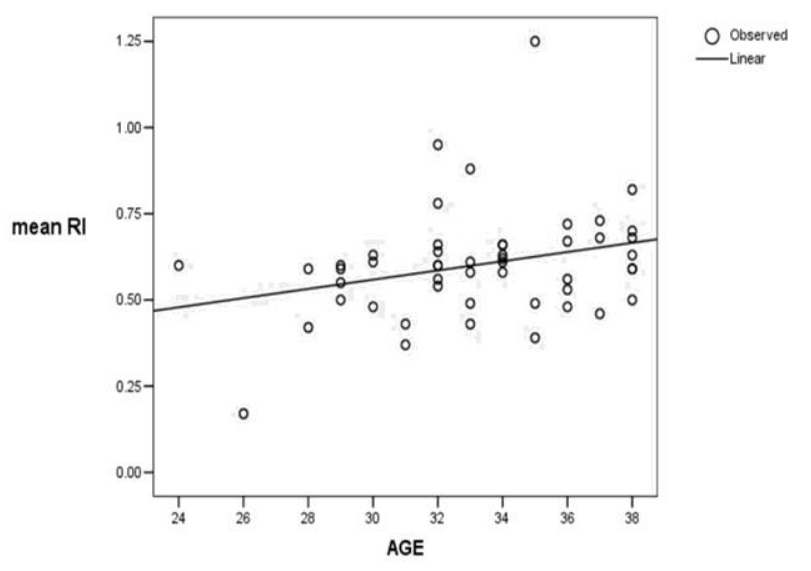

Figure 1: Correlation of age with ovarian stromal blood flow (resistance index - RI). 
The ovarian stromal blood flow was found to be negatively correlated with age. As depicted in figure 1 and 2 , both RI $(\mathrm{p}=0.02)$ and PI $(\mathrm{p}=0.01)$ were found to be significantly correlated with age.

As serum FSH level is considered to be an independent marker of ovarian reserve, we assessed whether any correlation existed between the ovarian stromal blood flow and the S.FSH level in a subject. There was however no such correlation found in this study. The subjects with abnormal S.FSH levels were excluded from the study; this probably explains the lack of any correlation between the ovarian stromal blood flow and the S.FSH level in this study.

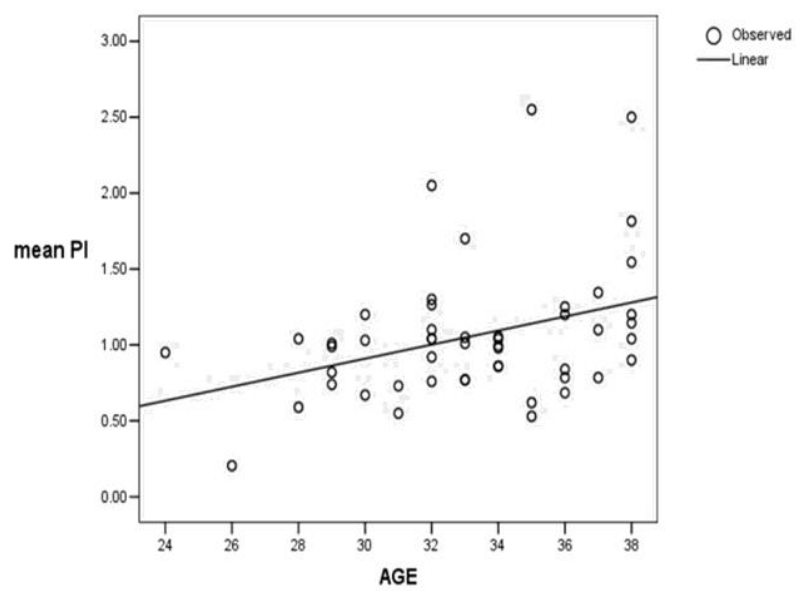

Figure 2: Correlation of age with ovarian stromal blood flow (pulsatility index - PI).

The number of follicles formed in a subject after the controlled stimulation with gonadotropins was found to be negatively correlated with the ovarian stromal RI and PI (table 2).

Table 2: Correlation of stroma blood flow and ovarian response.

\begin{tabular}{|lll|}
\hline \multirow{2}{*}{ Mean RI } & $\begin{array}{l}\text { Total number of follicles developed } \\
\text { after COH }\end{array}$ \\
\hline \multirow{2}{*}{ Mean PI } & $\begin{array}{l}\text { Correlation } \\
\text { coefficient }\end{array}$ & -0.204 \\
\cline { 2 - 3 } & $\begin{array}{ll}\text { P value } \\
\text { Correlation } \\
\text { coefficient }\end{array}$ & 0.15 \\
\cline { 2 - 3 } & P value & -0.292 \\
\hline
\end{tabular}

\section{DISCUSSION}

The probability for success with IVF relates to several factors, many of which are unfortunately not known until the treatment cycle is well underway or even nearing completion. Though a number of methods to measure ovarian reserve have been reported, no single method has been described which predicts future fecundability or provides prognostic information regarding the likelihood of successful treatment in infertile women.

This study was designed to evaluate the role of ovarian blood flow in predicting ovarian responsiveness in patients undergoing IVF. The main objective behind the study was to provide a non-invasive and cost-effective prognostic factor of IVF outcome. In the present study, a significant negative correlation was found between the ovarian stromal blood flow and age. As the age advanced, the mean ovarian stromal blood flow decreased. The relation between the increasing chronological age and decreasing fertility has been proven beyond doubt; however the mechanism of this decrease in fertility has always been a matter of debate. The observations of the present study may provide one of the mechanisms responsible for this decrease in fertility. $\mathrm{Ng}$ EHY et al, in their analysis of 119 women with proven fertility found no effect of age on mean Peak Systolic Velocity (PSV) of ovarian stromal blood flow calculated by Colour Doppler. ${ }^{5}$ However, with the advent of Power Doppler, the same authors reanalysed the effect of increasing age on ovarian blood flow in women with proven fertility. ${ }^{6}$ They observed that age was negatively correlated with ovarian flow index (FI), vascularization index (VI) as well as vascularization flow index (VFI). The rate of decline of total ovarian VI was found to be $0.18 \%$. However, statistically significant reduction in ovarian stromal blood flow occurred only in women $\geq 41$ years of age.

Zaidi et al were the first to show that there was a relation between ovarian stromal blood flow velocity and ovarian follicular response. ${ }^{7}$ However, the patients had not undergone pituitary suppression in their study and hence, the clinical utility in predicting IVF outcome on the basis of ovarian stromal blood flow remained doubtful. Engmann et al suggested an association between ovarian stromal blood flow PSV after pituitary suppression and the number of oocytes retrieved. ${ }^{8} \mathrm{NgEHY}$ et al opined differently and demonstrated in their study that ovarian stromal blood flow indices measured by 2D Power Doppler ultrasound had no predictive value for the ovarian response. ${ }^{9}$ In the present study, the ovarian responsiveness, in terms of the number of oocytes retrieved, was found to be correlated with the ovarian stromal blood flow. The correlation between the ovarian stromal blood flow and the ovarian responsiveness, demonstrated by this study, is not surprising because adequate ovarian blood flow is a prerequisite for normal ovarian function. Stromal blood flow may therefore become one of the baseline parameters available before ovarian stimulation to predict IVF outcome.

Funding: No funding sources

Conflict of interest: None declared

Ethical approval: Note required 


\section{REFERENCES}

1. Adamson D, Lancaster P, de Mouzon J, Nygren KG, Zegers Hochschild F. World collaborative report on assisted reproductive technology, 1998. In DL Healy, GT Kovacs, R McLachlan, O Rodriguez-Armas (eds), Reproductive medicine in the twenty first century. The Parthenon Publishing Group Ltd., UK. 2001:209-19.

2. Chang MY, Chiang CH, Hsieh TT, Soong YK, Hsu $\mathrm{KH}$. Use of the antral follicle count to predict the outcome of assisted reproductive technologies. 1998;69:505-10.

3. Ng EHY, Tang OS, Ho PC. The significance of the number of antral follicles prior to stimulation in predicting ovarian response in an In-vitro fertilization programme. Hum Reprod. 2000;15:1937-42.

4. Hsieh YY, Chang CC, Tsai HD. Antral follicle counting in predicting the retrieved oocyte number after ovarian hyperstimulation. J Assist Reprod Genet. 2001;18:320-4.

5. Ng EHY, Yeung WSB, Fong DYT, Ho PC. Effect of age on hormonal and ultrasound markers of ovarian reserve in Chinese women with proven fertility. Hum Reprod. 2003;18:2169-74.
6. Ng EHY, Chan CCW, Yeung WSB, Ho PC. Effect of age on ovarian stromal flow measured by three dimensional ultrasound with Power Doppler in Chinese women with proven fertility. Hum Reprod. 2004;19:2132-37.

7. Zaidi J, Barber J, Kyei-mensah A, Bekir J, Campbell S, Tan SL. Relationship of ovarian stromal blood flow at the baseline ultrasound scan to subsequent follicular response in an in vitro fertilization program. Obstet Gynecol. 1996;88:779-84.

8. Engmann L, Sladkevicius P, Agrawal R, Bekir JS, Campbell S, Tan SL. Value of ovarian stromal blood flow velocity measurement after pituitary suppression in the prediction of ovarian responsiveness and outcome of in vitro fertilization treatment. 1999;71:22-9.

9. NgEHY, Oi Shan Tang, Carina Chi Wai Chan, Pak Chung Ho. Ovarian stromal blood flow on the prediction of ovarian response during in vitro fertilization treatment. Hum Reprod. 2005;20:314751.

Cite this article as: Arora A, Gainder S, Dhaliwal LK, Suri V. Clinical significance of ovarian stromal blood flow in assessment of ovarian response in stimulated cycle for in vitro fertilization. Int J Reprod Contracept Obstet Gynecol 2015;4:1380-3. 